\title{
Leaf to panicle ratio (LPR): a new physiological trait indicative of source and sink relation in japonica rice based on deep learning
}

\author{
Zongfeng Yang ${ }^{1}$, Shang Gao², Feng Xiao' ${ }^{1}$ Ganghua Li', Yangfeng Ding ${ }^{1}$, Qinghua Guo ${ }^{2 *}$, Matthew J. Paul ${ }^{3}$ \\ and Zhenghui Liu ${ }^{1,4^{*}}$ (D)
}

\begin{abstract}
Background: Identification and characterization of new traits with sound physiological foundation is essential for crop breeding and production management. Deep learning has been widely used in image data analysis to explore spatial and temporal information on crop growth and development, thus strengthening the power of identification of physiological traits. Taking the advantage of deep learning, this study aims to develop a novel trait of canopy structure that integrate source and sink in japonica rice.

Results: We applied a deep learning approach to accurately segment leaf and panicle, and subsequently developed the procedure of GvCrop to calculate the leaf to panicle ratio (LPR) of rice canopy during grain filling stage. Images of training dataset were captured in the field experiments, with large variations in camera shooting angle, the elevation and the azimuth angles of the sun, rice genotype, and plant phenological stages. Accurately labeled by manually annotating the panicle and leaf regions, the resulting dataset were used to train FPN-Mask (Feature Pyramid Network Mask) models, consisting of a backbone network and a task-specific sub-network. The model with the highest accuracy was then selected to check the variations in LPR among 192 rice germplasms and among agronomical practices. Despite the challenging field conditions, FPN-Mask models achieved a high detection accuracy, with Pixel Accuracy being 0.99 for panicles and 0.98 for leaves. The calculated LPR displayed large spatial and temporal variations as well as genotypic differences. In addition, it was responsive to agronomical practices such as nitrogen fertilization and spraying of plant growth regulators.
\end{abstract}

Conclusion: Deep learning technique can achieve high accuracy in simultaneous detection of panicle and leaf data from complex rice field images. The proposed FPN-Mask model is applicable to detect and quantify crop performance under field conditions. The newly identified trait of LPR should provide a high throughput protocol for breeders to select superior rice cultivars as well as for agronomists to precisely manage field crops that have a good balance of source and sink.

Keywords: Plant phenotyping, Leaf and panicle detection, Deep learning, Physiological trait, Leaf to panicle ratio $(L P R)$, Japonica rice

\footnotetext{
*Correspondence: qguo@ibcas.ac.cn; liuzh@njau.edu.cn

${ }^{1}$ College of Agriculture, Nanjing Agricultural University, Nanjing 210095, China

${ }^{2}$ State Key Laboratory of Vegetation and Environmental Change, Institute of Botany, Chinese Academy of Sciences, Beijing 100093, China

Full list of author information is available at the end of the article
}

\section{Background}

Sustainable improvement in crop production is crucial for supporting the demand from an increasing global population, particularly considering that there are $821 \mathrm{M}$ people who lack sufficient food to support their daily lives [1]. Recent technological advances in genome biology

c) The Author(s) 2020. This article is licensed under a Creative Commons Attribution 4.0 International License, which permits use, sharing, adaptation, distribution and reproduction in any medium or format, as long as you give appropriate credit to the original author(s) and the source, provide a link to the Creative Commons licence, and indicate if changes were made. The images or other third party material in this article are included in the article's Creative Commons licence, unless indicated otherwise in a credit line to the material. If material is not included in the article's Creative Commons licence and your intended use is not permitted by statutory regulation or exceeds the permitted use, you will need to obtain permission directly from the copyright holder. To view a copy of this licence, visit http://creativecommons.org/licenses/by/4.0/. The Creative Commons Public Domain Dedication waiver (http://creativecommons.org/publicdomain/zero/1.0/) applies to the data made available in this article, unless otherwise stated in a credit line to the data. 
like next-generation sequencing, genome editing and genomic selection have paved the way for crop breeders to identify, characterize, transfer, or modify the genes responsible for grain yield or quality traits in a rapid and precise way [2]. However, there is a huge gap between the fundamental plant sciences and the applied science of crop breeding, as reflected by the limited understanding of the link between genotype and phenotype. Crop physiology is a key interface between the genome and the plant phenotype, and thus is indispensable for hastening crop improvement [3]. Accordingly, physiological breeding, a methodology for selection of physiological traits such as canopy temperature, carbon isotope discrimination, and stomatal conductance, was proposed. This approach has advantages over conventional breeding such as in water stressed Australian environments and in heat and drought stressed conditions of the International Wheat Improvement Network [4].

Chemically, cereal grain yield consists of photosynthetic assimilates first produced in the leaf source organs which are translocated to the sink organ of grain. Therefore, source and sink relations, the core concept of crop physiology, is the critical factor dominating crop yield formation. Improving the source activity of leaf photosynthesis to harness light irradiation more efficiently is one of the major targets of crop breeding. In rice, ideotypes have long been pursued by breeders, resulting in several successfully implemented theories such as the New Plant Type, Super Rice, and Ideal Plant Architecture [5-7]. One common future shared by these new plant types is the emphasis on leaf erectness, especially the top three leaves, which is supposed to be essential for improving source activity. However, some of the main cultivars with this ideotype had problems of incomplete filling of inferior grains, especially for those with large numbers of grains, indicating the importance of optimization of the source-sink ratio $[8,9]$.

In addition to storing photosynthetic assimilates from leaves, sink organs like glumes and awns have photosynthetic activity. Cumulative evidence favors the sizable contribution of spike or panicle to grain filling in terms of providing carbohydrates as well as nitrogen $(\mathrm{N})$, magnesium, and zinc $[10,11]$. In wheat and barley, contribution of spikes to grain filling has a range of $10 \%$ to $76 \%$ [12]. In rice, gross photosynthetic rate of the panicle is $30 \%$ of that of the flag leaf, and it was estimated that panicle photosynthesis contributed $20 \%$ to $30 \%$ of the dry matter in grain [13]. Thus light interception of the ear or panicle should be integrated into the breeding programmes aiming for source-sink balance.

Technical advances in high throughput field phenotyping on a breeding scale in realistic field environments have strengthened the power of physiological breeding [4]. Concurrently, methods for data mining of the big data acquired by various phenotyping platforms are developed. Among them, deep learning has been widely used in image data analysis to explore spatial and temporal information concerning crop growth and development [14]. Leaf area and number indicate the photosynthetic capacity of the crop canopy, and the precise segmentation and counting of leaves has been one of the objectives of image processing. Studies have resulted in robust methodology of deep learning for quantifying leaf number from 2D images [15] and 3D images [16-18], providing effective tools for growth estimation and yield prediction of crop plants. Spike (wheat) or panicle (rice) number per square meter is the key component of cereal grain yield. Numerous attempts have been made to segment and count this reproductive organ accurately in rice [19-21] and wheat [22-24]. Collectively, these robust, low-cost and efficient methods to assess the number of economic organs are of high relevance for phenotyping efforts towards increases in cereal grain yield. However, to our knowledge, method development is still necessary to simultaneously extract both leaf and panicle from the background of a field crop population, as required by the breeder to adopt physiological strategies to balance source and sink.

In this study, we applied a deep learning approach to accurately extract leaf and panicle image data and subsequently calculate the leaf to panicle ratio (LPR) of rice populations during grain filling stage. Of note, the LPR is proposed as a proximate estimation of the distribution of light interception between leaf and panicle, with an assumption that the light captured by the camera is the sunlight directly reflected by the leaf or panicle. Images of training dataset were captured in the field experiments, with large variations in camera shooting angle, the elevation angle and the azimuth angle of the sun, rice genotype, and plant phenological stages. Accurately labeled by manually annotating all the panicle and leaf regions, the resulting dataset were used to train models of FPNMask (Feature Pyramid Network Mask) [25], consisting of a backbone network and a task-specific sub-network. The model with the highest accuracy was then selected to study the variations in LPR among 192 japonica rice germplasms and among agronomical practices. Our aim was to provide a high throughput protocol and new physiological trait for breeders to select superior rice cultivars as well as for agronomists to precisely manage field crops that have a good balance of source and sink.

\section{Methods}

We explored an end-to-end semantic segmentation method to label each pixel as panicle, leaf or background automatically under natural field conditions, and then 
generated the leaf to panicle ratio (LPR) by division of the number of pixels assigned for each class in each field image. Figure 1 shows the overall work-flow of this method, including two parts. Part 1 is the offline training workflow, with the aim of building a deep learning network called FPN-Mask to segment panicle and leaf from field RGB images. Part 2 is the procedure of GvCrop to develop a software system for calculating LPR.

\section{Experimental setup}

In 2018, plots of ongoing field experiments at Danyang $\left(31^{\circ} 54^{\prime} 31^{\prime \prime} \mathrm{N}, 119^{\circ} 28^{\prime} 21^{\prime \prime} \mathrm{E}\right)$, Jiangsu Province, China were selected to take pictures for the training dataset. Of note, these experiments were not specially designed for a phenotyping study. In brief, plant materials of these experiments were highly diverse in genotypic variation, containing seven main japonica cultivars of Jiangsu and 195 mutants with contrasting agronomical traits as reported by Abacar et al. [26]. Further, the seven cultivars had two sowing dates, resulting in obviously different phenotypes for a certain genotype. Thus the diversity in plant architecture and canopy structure of tested materials can provide as many kinds of phenotypes as possible for image analysis.

In 2019, three experiments were conducted to test and apply the proposed FPN-Mask model. (1) Genotypic variations in LPR. A total of 192 mutants were investigated. The plot area was $2.4 \mathrm{~m} \times 1.4 \mathrm{~m}$ with a row spacing of $30 \mathrm{~cm}$ and plant spacing $20 \mathrm{~cm}$. Nitrogen, phosphate $\left(\mathrm{P}_{2} \mathrm{O}_{5}\right)$ and potassium $\left(\mathrm{K}_{2} \mathrm{O}\right)$ fertilizers were applied at a rate of $240 \mathrm{~kg} \mathrm{ha}^{-1}, 120 \mathrm{~kg} \mathrm{ha}^{-1}$ and $192 \mathrm{~kg} \mathrm{ha}^{-1}$, respectively, and were equally separated into basic fertilizers (before transplanting) and topdressing (at 4th leaf age in reverse order). (2) $\mathrm{N}$ fertilization effects on LPR. A japonica rice cultivar, Wuyunjing 30, was selected for field experiments with a randomized complete-block design. It had three replications, with a plot area of $2.4 \mathrm{~m} \times 1.4 \mathrm{~m}$. Total $\mathrm{N}$ fertilizer was $240 \mathrm{~kg} \mathrm{ha}^{-1} \mathrm{~N}$, and two $\mathrm{N}$ fertilization modes with different base/topdressing ratios were applied: (1) N5-5: base/topdressing, 5/5; (2) N10-0: base/ topdressing, 10/0. (3) Regulation of plant growth regulators on LPR. Solutions of $100 \mu \mathrm{M}$ gibberellin, $100 \mu \mathrm{M}$ uniconazole, $25 \mu \mathrm{M}$ 2, 4-epibrassinolide, $25 \mu \mathrm{M}$ brassinazole as well as the control, water, were prepared in distilled water with $0.5 \%$ TWEEN-20. One cultivar, Ningjing 8 , from the $\mathrm{N}$ treatment was used as material. Spraying was conducted at the rate of $500 \mathrm{~mL} \mathrm{~m}^{-2}$ after sunset, with three times starting at booting stage on August 22 and with a 2-day interval.

In addition, a dynamic canopy light interception simulating device (DCLISD) was used to capture images that reflect the sun's perspective (Fig. 2). The bottom part of it consists of four pillars with wheels and the upper is comprised of two arches consolidated by two steel pipes, and a moveable rail for mounting the RGB camera. The sun's trajectory is simulated by two angles, the elevation

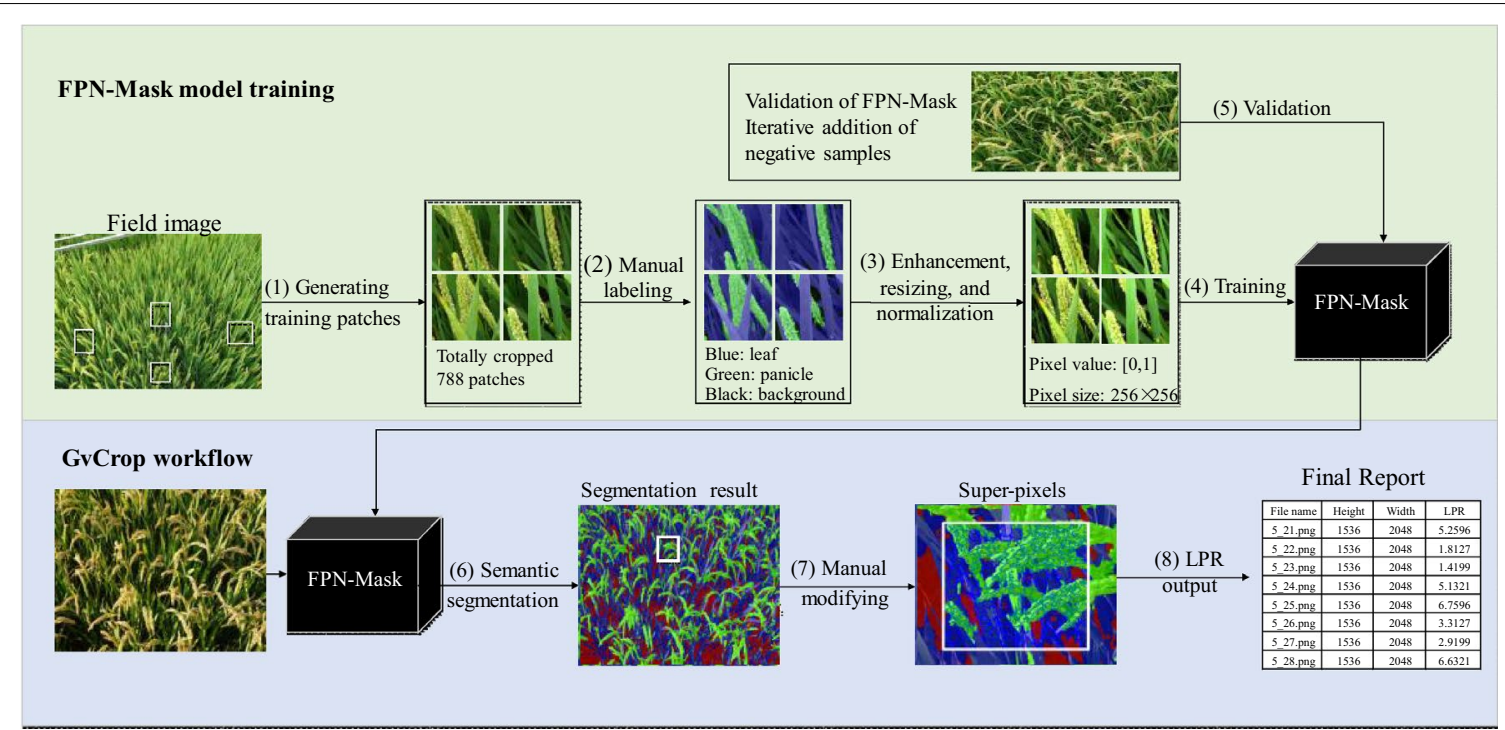

Fig. 1 The overall work-flow of panicle-leaf quantification. The upper shows the training procedure of the FPN-Mask model implemented in this study. The bottom depicts the GvCrop working procedure to calculate the LPR (leaf to panicle ratio). (1) Generating 1896 patches by random manual cutting. (2) Manual labelling of every pixel to panicle, leaf and background. (3) Brightness enhancement of patches, normalization to [0, 1] and resizing to $256 \times 256$ pixels. (4) Training the FPN-Mask model. (5) Daily validation of FPN-Mask with field images and iterative addition of negative samples. (6) Integration of the saved model to semantic segmentation of field images by GvCrop. (7) Manual modification of the predicted result by super-pixel segmentation method integrated in GvCrop 
a

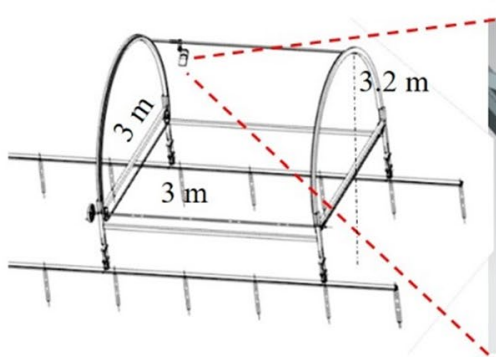

b

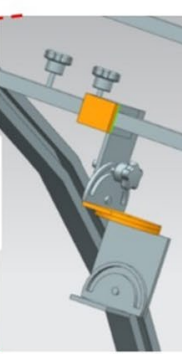

$\mathbf{c}$

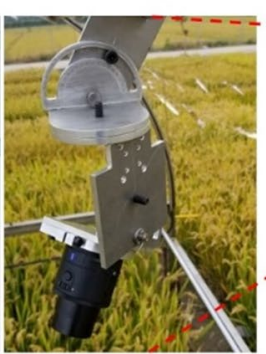

d

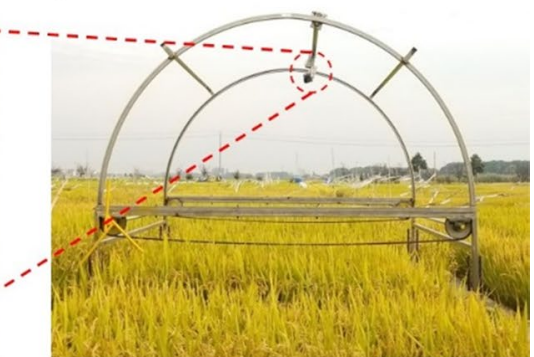

Fig. 2 Dynamic canopy light interception simulating device (DCLISD). a sketch design of DCLISD. $\mathbf{b}$ sketch design of unit for simulating the sun's trajectory by two angles, the elevation and the azimuth angle. c RGB camera mounted on the simulation unit; $\mathbf{d}$ working scene of DCLISD

angle and the azimuth angle, calculated according to the latitude, longitude, as well as the growth periods at the experimental site.

\section{Image acquisition}

Images of the training dataset were captured in the field experiments in 2018, reflecting the large variations in camera shooting angle, the elevation angle and the azimuth angle of the sun, rice genotype [27], and plant phenological stages (Fig. 3). Images for validation and application of the proposed model were acquired in 2019. For the three treatments of genotypes, $\mathrm{N}$ fertilization, and spraying, an angle of $40^{\circ}$ was selected for the tripod. The height of the camera (Canon EOS 750D, 24.2 megapixels) was $167.1 \mathrm{~cm}$, the average height of a Chinese adult, and the distance between the central point of the target area and vertical projection of the camera on the ground was $90 \mathrm{~cm}$. The camera settings were as follows: focal length,
$18 \mathrm{~mm}$; aperture, automatic; ISO, automatic; and exposure time, automatic. In the experiment with DCLISD, the camera model was SONY DSC-QX100, with settings were as follows: focal length, $10 \mathrm{~mm}$; aperture, automatic; ISO, automatic; and exposure time, automatic.

\section{Dataset preparation Training dataset}

Taking into consideration camera angle, solar angle, panicle type and growth stage (Fig. 3), we prepared a training dataset with 360 representative images from the 2018 dataset (Additional file 1: Table S1). The GG (Green panicle with Green leaf) growth stage, YG (Yellow panicle with Green leaf) growth stage and YY (Yellow panicle with Yellow leaf) growth stage were represented by 113, 104, and 143 images, respectively. Figure 1(1-3) shows the preparation of training data. Considering that the original size of these field images is as large as

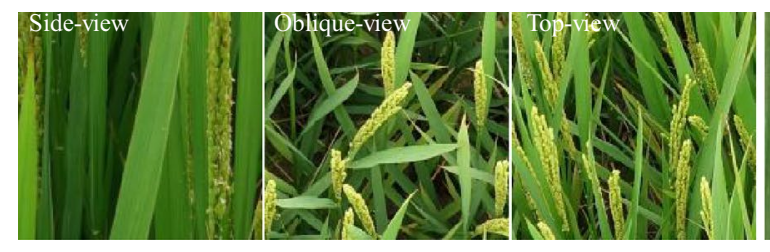

a Camera shooting angles

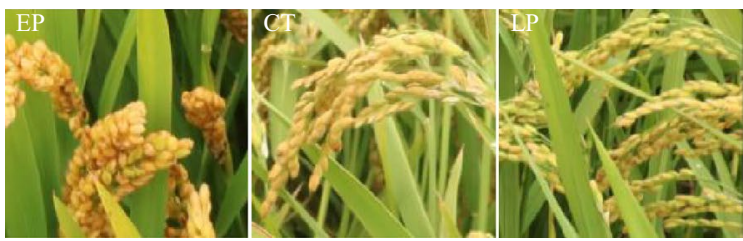

c Panicle types

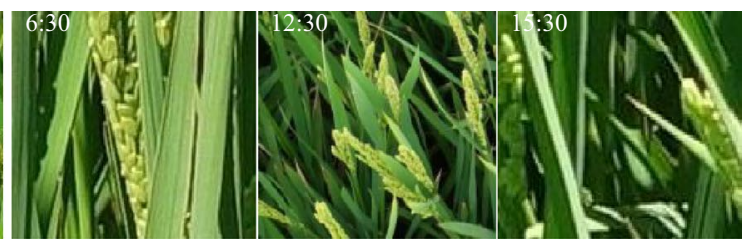

b Solar angles

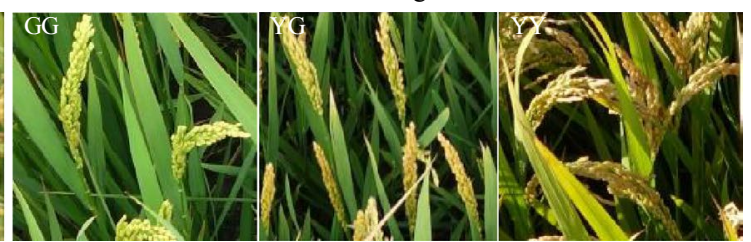

d Phenological stages

Fig. 3 Images selected as training dataset under different field conditions in 2018. a camera shooting angles, including side-view (180 ${ }^{\circ}$, oblique-view $\left(45^{\circ}\right)$ and top-view $\left(90^{\circ}\right)$; b solar angles in the morning (5:30 a.m.-10:00 a.m.), at noon (11:00 a.m.-13:00 p.m.) and in the afternoon(15:30 p.m.-18:30 p.m.); c cultivars with three panicle types, EP (erect panicle), CT (chicken toe), and LP (loose panicle), according the classification by Zhang et al. [27]; d phenological stages, GG (Green panicle with Green leaf) at early, YG (Yellow panicle with Green leaf) at middle, and YY (Yellow panicle with Yellow leaf) at late stage of grain filling 
$4864 \times 3648$ pixels, they were cropped to a size between $[150,150]$ and $[600,600]$ using the Paint.Net software. After obtaining these patches, we labeled pixels of each patch as panicle, leaf and background manually using the Fluid Mask software. Finally, a total of 1896 representative patches were selected as the final training sample set. Among them, 1210 samples were added continuously during the late daily tests of the model. Further, to increase the diversity of the training dataset and avoid overfitting, we performed basic data enhancements to the training set, including random horizontal/vertical flips, rotations by $90^{\circ}$, and histogram equalizations. To reduce illumination effects, we performed random brightness enhancements on the image. All the input images were resized to $256 \times 256$ pixels. And for a faster and more stable training model, all the input images were normalized to $[0,1][28,29]$.

\section{Testing dataset}

We divided all 2018 collected images into three groups based on rice growth stage. From each group, we randomly selected 35 testing images and finally selected 105 images as the testing dataset (Additional file 1: Table S2). These selected testing images included extraneous objects, such as tracks, chains, neighbor plots and color-charts.

\section{Network structure}

In this study, we proposed a deep learning-based method for rice panicle segmentation, called FPN-Mask. The method consists of a backbone network and a Task-specific subnetwork. The Feature Pyramid Network (FPN) [25] was selected as the backbone network for extracting features over the entire input data. Originally designed for object detection, it has the advantages of extracting a multi-level feature pyramid from an input image with a single scale. The subnetwork is referenced from the
Unified Perceptual Parsing Network [30], which performs semantic segmentation based on the output of the backbone network (Fig. 4).

Backbone network for feature extraction: The FPN [25] is a standard feature extractor with a top-down architecture and lateral connections. The top-down architecture is based on Residual Networks (ResNet) [31], with four stages denoted as $\mathrm{C}_{2}, \mathrm{C}_{3}, \mathrm{C}_{4}$ and $\mathrm{C}_{5}$, respectively. We denoted the last feature map of each stage in ResNet as $\left\{C_{2}, C_{3}, C_{4}, C_{5}\right\}$. In our backbone network, we removed the global max pooling layer before $\mathrm{C}_{2}$, because it will drop out semantic information. Therefore, the rates of each stage $\left\{\mathrm{C}_{2}, \mathrm{C}_{3}, \mathrm{C}_{4}, \mathrm{C}_{5}\right\}$ were down-sampled from $\{4,8,16,32\}$ to $\{1,2,4,8\}$. The down-sampling rates of the feature maps derived by FPN $\left\{\mathrm{P}_{2}, \mathrm{P}_{3}, \mathrm{P}_{4}, \mathrm{P}_{5}\right\}$ were $\{1,2$, $4,8\}$, respectively, meaning that the size of $\mathrm{P}_{2}$ is the same as the original image size of $256 \times 256$, while that of $\mathrm{P}_{5}$ is $32 \times 32$. The number of feature maps output for each stage in ResNet is equal to 32.

Subnetwork for semantic segmentation: the subnetwork is based on the multi-level features extracted from the backbone network. Each level of the features will be fused together as an input feature map for semantic segmentation, which has been proved to outperform that only using the highest resolution feature map [30, 32]. To up-sample the low-level feature maps $\left\{\mathrm{p}_{3}, \mathrm{p}_{4}, \mathrm{p}_{5}\right\}$ to get the same size feature as the original image, we directly adopted the bilinear interpolation layer instead of the time-consuming deconvolution layer, and attached a $1 \times 1$ convolution layer followed by each interpolation layer to refine the interpolation result. After up-sampling, different levels of the features were concatenated as the final semantic feature. The concatenated multi-level features were convoluted by a $3 \times 3$ convolution layer to refine the result and a $1 \times 1$ convolution layer to reduce the channel dimensions. The $3 \times 3$ convolution layer was attached to a batch normal layer and a relu layer. Finally,

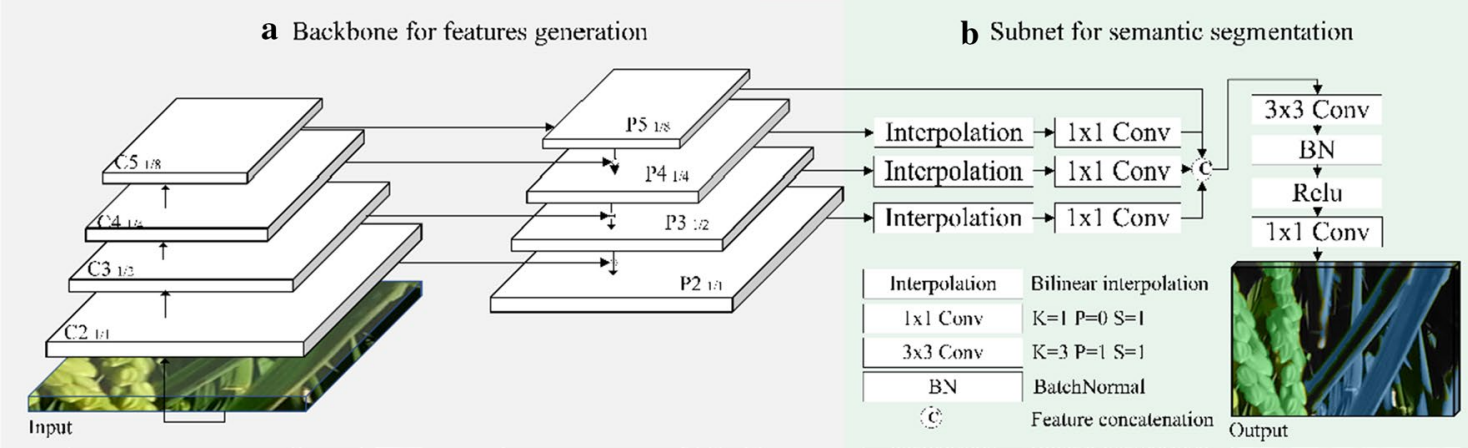

Fig. 4 Network architecture. The input layer is a normalized patch, shown in color images. The output consists of the segmented pixels in different colors: green (panicle), blue (leaf), and black (background) 
we obtained a 3-channel semantic segmentation result, representing background, leaf and panicle, respectively.

\section{Loss function for semantic segmentation}

The cross-entropy loss function is a standard classification method [33]. In practical application, due to the uneven number of pixels from different categories, the loss calculated by the cross-entropy loss function is not realistic [34]. Thus we used the focal loss, which is specifically designed to solve the imbalance problem by focusing on the more difficult classification locations through changing the weight of different categories [34].

\section{Training}

We experimented with ResNet-18 as the FPN backbone. All convolutional layers were initialized as in $\mathrm{He}$ et al. [35]. Batch layers were simply initialized with bias $b=0$ and weight $\sigma=0$. The mini-batch size was 24, optimization was based on the Adam method, and the training process lasted for 7 days with the base learning rate of 0.001 . All the training were conducted on a high-performance computer with Intel $3.50 \mathrm{GHz}$ processor and 128 GB of memory. Two NVIDIA 1080 GeForce graphics processing unit (GPU) has a $12 \mathrm{~GB}$ memory used to accelerate the training of our model.

During training, we tested the model performance with all the collected images and selected supplementary training samples for the images that did not perform well to make sure that the training samples covered all the cases of the 6 GB images obtained in 2018 (except the 90 testing images). Sixty images generating 302 patches were added as supplementary training samples, being about 40 samples per day. The performance standards (good or bad) were judged by observation. The training continued until the testing performance of all images visually met the accuracy requirements and the loss function curve was smooth without fluctuations.

\section{PostProcess}

Although a deep network is well suited for processing semantic segmentation problems, errors can not be completely avoided. To lessen the influence of segmentation errors, we further developed a tool, called GvCrop, for manually modifying the auto segmentation results. The software does not only integrate the pixel-wise segmentation method (Fig. 1(6)), but also integrates the ability to modify the segmentation results by human interaction (Fig. 1(7)). Because pixel-level labelling of the wrong location is time consuming, processing the image regions with homogeneous characteristics instead of single pixels can help us accelerate the manual labelling speed (Fig. 1(7)). According to the image color space and boundary cues, we used the gSLICr algorithm to group pixels into perceptually homogeneous regions [36]. gSLICr is the Simple Linear Iterative Clustering (SLIC) implemented on GPU using the NVIDIA CUDA framework, $83 \times$ faster than the SLIC CPU implementation [37]. The gSLICr has three parameters: S, C and N. $\mathrm{S}$ stands for super pixel size, $\mathrm{C}$ the compact coefficient degree, and $\mathrm{N}$ the number of iterations. In this study, $\mathrm{S}$ was set to $15, \mathrm{C}$ to 0.2 , and $\mathrm{N}$ to 50 . After super pixel segmentation, users can modify the auto-segmentation results based on super pixels.

\section{Accuracy assessment}

To quantify the performance of the proposed method, we employed three standard metrics to quantify semantic segmentation tasks. (1) Pixel Accuracy (P.A.) indicates the proportion of correctly classified pixels to the total number of pixels. (2) IoU indicates the Intersection-overunion between the ground truth and the predicted pixels, averaged over all classes. (3) Area under the receiver operating characteristic (ROC) curve [38].

$$
\begin{aligned}
& \text { P.A. }=\frac{\sum_{i}^{n} p_{i i}}{\sum_{i=0}^{n} \sum_{j=0}^{n} p_{i j}}(1) \\
& \text { mIoU }=\frac{1}{n+1} \times \frac{\sum_{i}^{n} p_{i i}}{\sum_{i=0}^{n} p_{i j}+\sum_{j=0}^{n} p_{j i}-p_{i i}}
\end{aligned}
$$

where $n$ is the number of classes, $p_{i j}$ is the number of pixels of class $i$ predicted to belong to class $j, p_{i i}$ is the true positive, $p_{i j}$ is the false negative, $p_{j i}$ is the false positive, $p_{j j}$ is the true negative.

\section{Calculation of leaf-panicle ratio (LPR)}

The software GrCrop was developed to calculate LPR based on the quantity of pixels contained in the leaf $(\mathrm{L})$ and panicle $(\mathrm{P})$ regions in an image and is calculated as: $\mathrm{LPR}=\mathrm{L} / \mathrm{P}$.

\section{Results}

\section{Accuracy verification}

The semantic segmentation of the 105 field images was assessed both visually (Fig. 5) and quantitatively (Table 1). In addition, we added extra datasets that include nonvegetated objects to evaluate the performance of our proposed method. Fifteen field images at 3 growing stages, 5 images per stage, were added to the testing dataset. The segmentation results using FPN-Mask segmentation method were shown in Additional file 1: Fig. S1, and the corresponding accuracy assessment results were presented in Table 1.

Figure 5 shows some examples of semantic segmentation results. Visual assessment suggested that the tested 


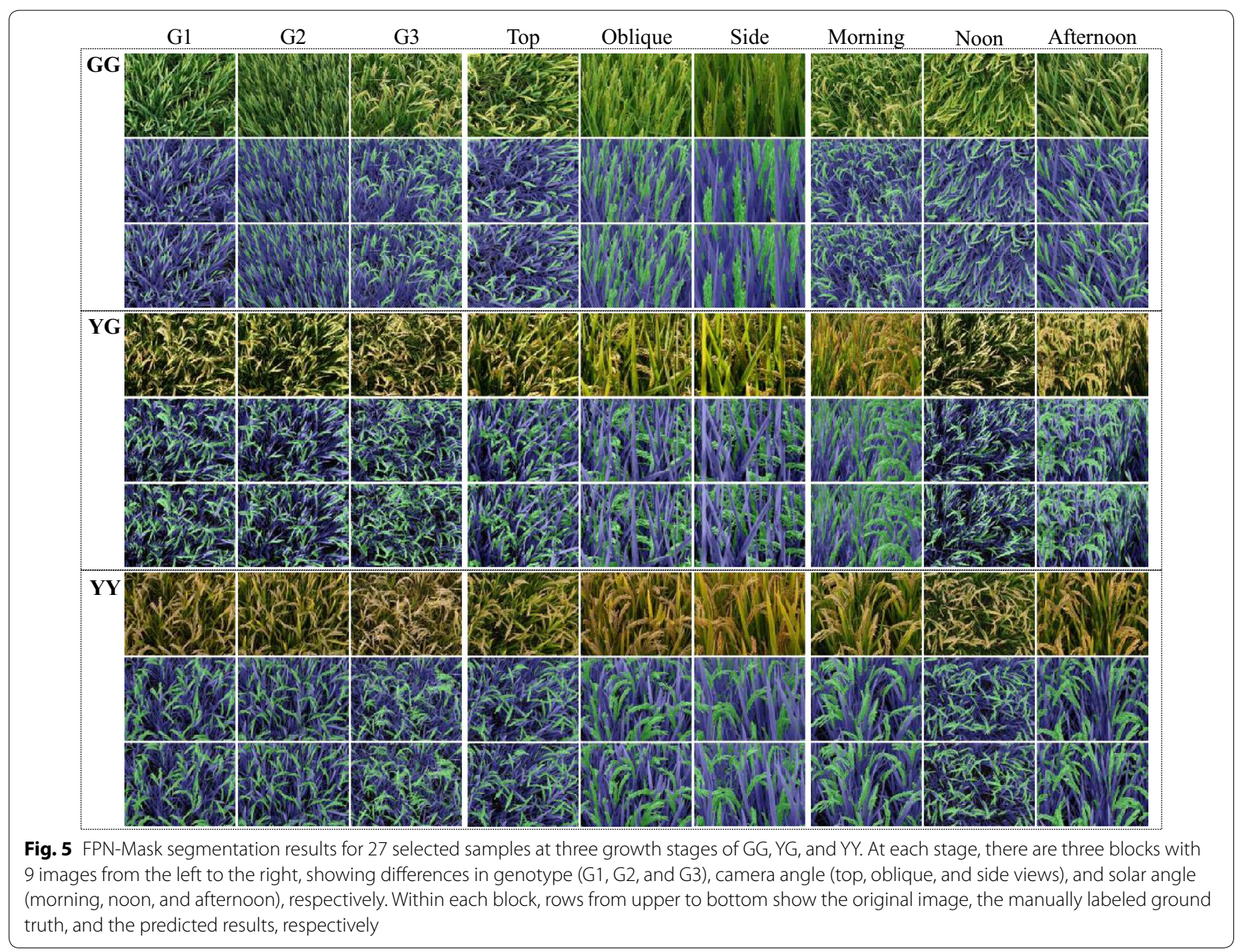

results and real data were very similar in different conditions. However, we still found some subtle segmentation errors: (1) The background and shadow pixels of the leaves were very similar visually, resulting in some shadow pixels being misclassified as background; (2) The segmentation was a little poor at the edges of the plant parts, with pixels at the junction between leaf and panicle being misclassified into error categories; (3) Some scattered small patches on the leaves were misclassified as panicle. The area under the ROC curve by using our model with the testing datasets were $0.95,0.94$ and 0.94 for GG, YG, and YY, respectively (Fig. 6).

Table 1 provides a quantitative evaluation of the complete test set, showing the high accuracy of all the testing images. Differences between images and growth stages were quite small. Additional file 1: Table S3 shows the model can reach $99 \%$ accuracy for panicles pixels, followed by leaf pixels ( $97.6 \%$ to $98.3 \%$ ), while the worse was for background pixels, ranging from 81.4 to $89.4 \%$.
The efficiency of a training model can also be described in terms of training data loss. Additional file 1: Fig. S2 exhibits that there was a rapid decline in loss over subsequent epochs of training, although the loss was initially high. To avoid overfitting and improve the robustness of our model, we iteratively added samples to the training dataset (Fig. 1(5)) and performed basic data enhancements randomly to the training set before putting it into the model.

\section{Verification and application of the FPN-Mask model}

The most important output of this FPN-Mask model is to estimate the distribution of light interception between leaf and panicle. Using GvCrop, we calculated the LPR values of the crop stand for various field experiments and detected large spatial and temporal variations as well as genotypic differences. Overall, these results suggest the feasibility of the model in detecting and quantifying crop performance under field conditions. 
Table 1 Accuracy assessments of the automatic leaf-panicle segmentation results

\begin{tabular}{|c|c|c|c|c|c|c|}
\hline \multirow[t]{2}{*}{ ID } & \multicolumn{2}{|l|}{ GG } & \multicolumn{2}{|l|}{ YG } & \multicolumn{2}{|l|}{ YY } \\
\hline & mloU & PA & mloU & PA & mloU & PA \\
\hline 1 & 0.861 & 0.937 & 0.846 & 0.912 & 0.900 & 0.950 \\
\hline 2 & 0.851 & 0.945 & 0.860 & 0.921 & 0.899 & 0.952 \\
\hline 3 & 0.840 & 0.946 & 0.830 & 0.902 & 0.866 & 0.933 \\
\hline 4 & 0.849 & 0.958 & 0.844 & 0.915 & 0.861 & 0.934 \\
\hline 5 & 0.842 & 0.959 & 0.845 & 0.919 & 0.854 & 0.936 \\
\hline 6 & 0.837 & 0.954 & 0.846 & 0.921 & 0.850 & 0.940 \\
\hline 7 & 0.833 & 0.954 & 0.850 & 0.927 & 0.856 & 0.944 \\
\hline 8 & 0.822 & 0.948 & 0.847 & 0.923 & 0.837 & 0.933 \\
\hline 9 & 0.825 & 0.950 & 0.847 & 0.924 & 0.842 & 0.936 \\
\hline 10 & 0.881 & 0.987 & 0.887 & 0.959 & 0.890 & 0.945 \\
\hline 11 & 0.869 & 0.984 & 0.864 & 0.943 & 0.874 & 0.931 \\
\hline 12 & 0.857 & 0.978 & 0.855 & 0.934 & 0.887 & 0.942 \\
\hline 13 & 0.846 & 0.979 & 0.845 & 0.925 & 0.889 & 0.941 \\
\hline 14 & 0.837 & 0.978 & 0.841 & 0.928 & 0.881 & 0.935 \\
\hline 15 & 0.847 & 0.976 & 0.851 & 0.936 & 0.880 & 0.938 \\
\hline 16 & 0.851 & 0.978 & 0.856 & 0.938 & 0.885 & 0.943 \\
\hline 17 & 0.852 & 0.978 & 0.860 & 0.942 & 0.872 & 0.937 \\
\hline 18 & 0.863 & 0.974 & 0.863 & 0.946 & 0.875 & 0.938 \\
\hline 19 & 0.825 & 0.962 & 0.867 & 0.948 & 0.880 & 0.942 \\
\hline 20 & 0.824 & 0.961 & 0.871 & 0.949 & 0.873 & 0.938 \\
\hline 21 & 0.824 & 0.961 & 0.871 & 0.950 & 0.871 & 0.938 \\
\hline 22 & 0.831 & 0.960 & 0.870 & 0.949 & 0.871 & 0.939 \\
\hline 23 & 0.838 & 0.959 & 0.868 & 0.947 & 0.874 & 0.941 \\
\hline 24 & 0.837 & 0.960 & 0.876 & 0.948 & 0.874 & 0.942 \\
\hline 25 & 0.836 & 0.961 & 0.880 & 0.948 & 0.874 & 0.942 \\
\hline 26 & 0.833 & 0.962 & 0.879 & 0.947 & 0.874 & 0.942 \\
\hline 27 & 0.833 & 0.963 & 0.877 & 0.947 & 0.872 & 0.940 \\
\hline 28 & 0.832 & 0.962 & 0.877 & 0.947 & 0.871 & 0.940 \\
\hline 29 & 0.831 & 0.962 & 0.869 & 0.942 & 0.870 & 0.941 \\
\hline 30 & 0.833 & 0.963 & 0.867 & 0.942 & 0.871 & 0.942 \\
\hline 31 & 0.889 & 0.966 & 0.931 & 0.974 & 0.934 & 0.986 \\
\hline 32 & 0.879 & 0.968 & 0.934 & 0.973 & 0.953 & 0.986 \\
\hline 33 & 0.901 & 0.970 & 0.934 & 0.973 & 0.960 & 0.985 \\
\hline 34 & 0.914 & 0.973 & 0.941 & 0.975 & 0.968 & 0.986 \\
\hline 35 & 0.912 & 0.972 & 0.939 & 0.974 & 0.976 & 0.989 \\
\hline Mean & 0.850 & 0.964 & 0.871 & 0.941 & 0.970 & 0.987 \\
\hline Min & 0.822 & 0.937 & 0.830 & 0.902 & 0.837 & 0.931 \\
\hline Max & 0.914 & 0.987 & 0.941 & 0.975 & 0.976 & 0.989 \\
\hline Std & 0.025 & 0.012 & 0.014 & 0.014 & 0.014 & 0.005 \\
\hline
\end{tabular}

GG green panicle with green leaf, $Y G$ yellow panicle with green leaf, $Y Y$ yellow panicle with yellow leaf, mloU mean intersection-over-union, $P A$ pixel accuracy, Min minimum, Max maximum, Std standard error of mean

Daily changes of LPR

LPR showed an obvious pattern of daily change, being higher after sunrise and before sunset but lower at noon (Fig. 7). The larger values of LPR in the morning or afternoon can be explained by the shading of leaves when the solar angle of incidence is lower.
Genotypic variations in LPR

Large genotypic differences in LPR were detected among the 192 mutants, ranging from 1.37 to 5.60 (Additional file 1: Table S4). As shown in Fig. 8, the six panicle types showed marked differences in LPR. Generally, cultivars with compact panicle (CP) had 


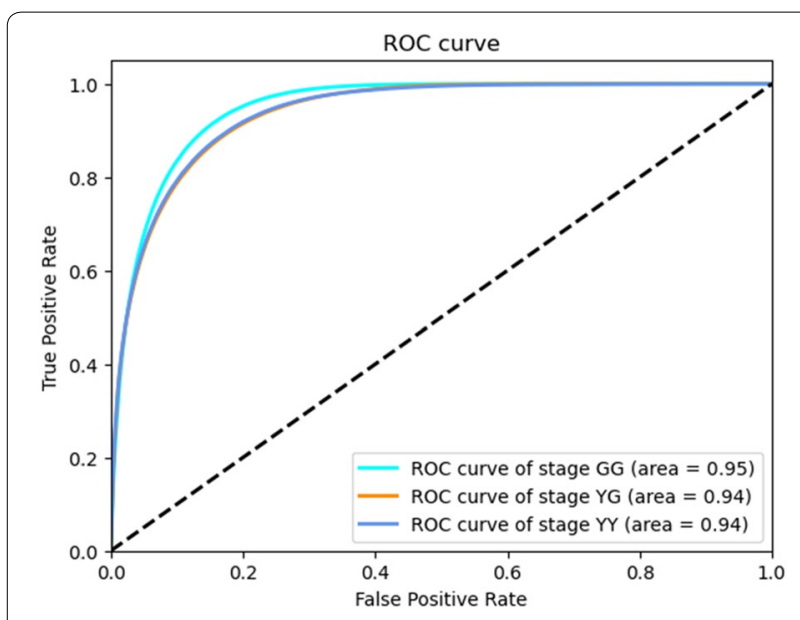

Fig. 6 ROC curves and the area under the ROC curves for test dataset at three growth stages of $G G, Y G$, and $Y Y$. ROC, receiver operating characteristic

the highest value, while those with loose panicle and awns (LPA) had the lowest. The former can be associated with the high density of spikelets on the panicle that caused smaller panicle area. The latter can be explained by the large panicle area due to sparse spikelets. Temporal variations of LPR were revealed showing a diminishing trend from the early stage to the late stage of grain filling. This means the relative area of leaf was reduced, as it is partly due to the increased area of panicle that changes its shape from erect and dense at early stage to loose and drooping at late stage.

\section{$N$ effect on $L P R$}

$\mathrm{N}$ fertilization mode exerted substantial influence on LPR. On average, $\mathrm{N}$ topdressing of the N5-5 increased LPR by 0.45 and 0.76 at middle and late stage, respectively, compared with N10-0 (Fig. 9). The promoting effect of $\mathrm{N}$ topdressing is associated with the elongation of flag leaf (Fig. 9). Similarly, LPR decreased gradually as grain filling progressed for both $\mathrm{N}$ treatments.

\section{Modification of LPR by plant growth regulators}

Plant growth regulators like brassinolide, brassinazole, gibberellin, and uniconazole obviously reshaped the canopy architecture (Fig. 10). The effects of these regulators agreed well with their well-documented phenotypes, for example, the drooping flag leaf caused by brassinolide spraying $[39,40]$ and the elongated upper internode caused by gibberellin [41]. More importantly, LPR can be either up-regulated or down-regulated by these regulators, depending on growth stages. As shown in Fig. 10, LPR at grain filling stage was increased by brassinazole and uniconazole, whereas reduced by brassinolide and gibberellin. In addition, the degree of increase or decrease depended on regulators, with uniconazole having the most significant influence.

\section{Discussion}

\section{Weakness of the methodology and improvement}

In this study, we built a robust and highly accurate deep learning network, FPN-Mask, which can easily segment panicle, leaf and background at a pixel level from a field RGB image. We also developed the GvCrop software, which not only included some basic image processing functions such as $\mathrm{I} / \mathrm{O}$, cut, rotation, zoom in/out, translation, but also integrated the above-mentioned auto

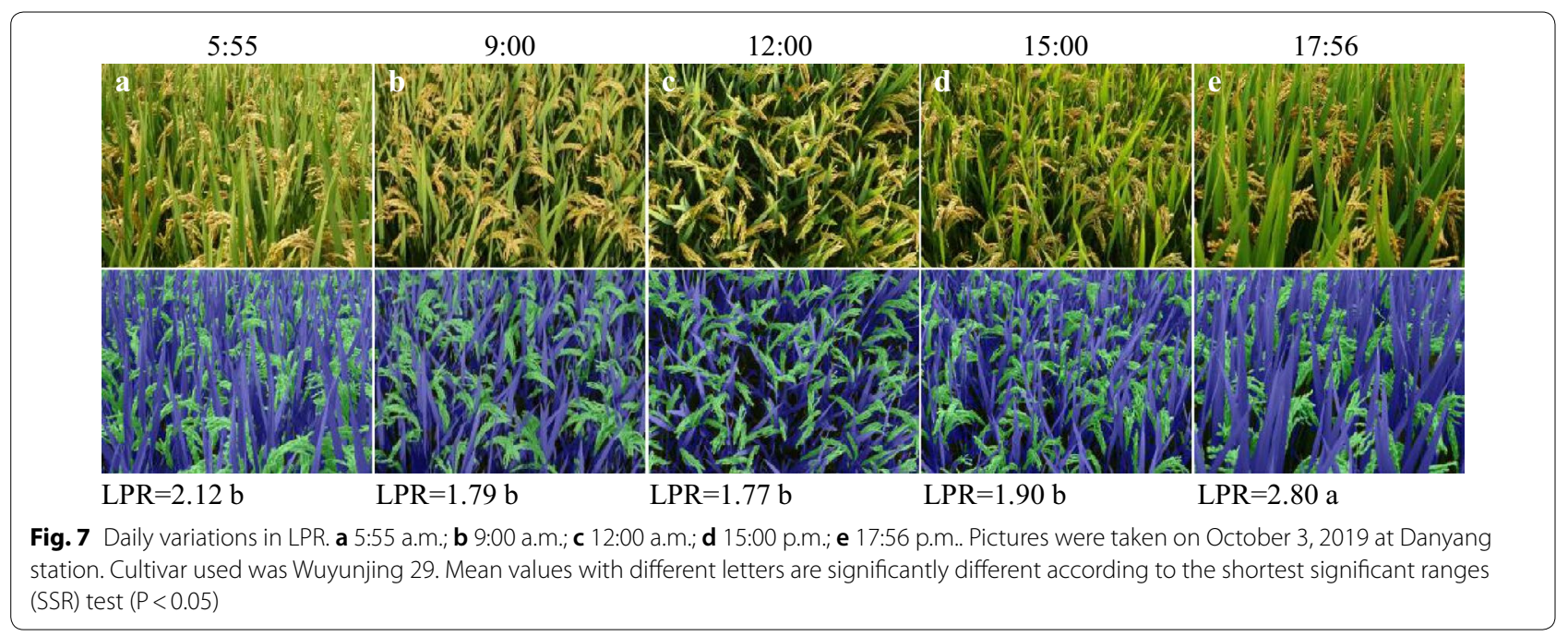



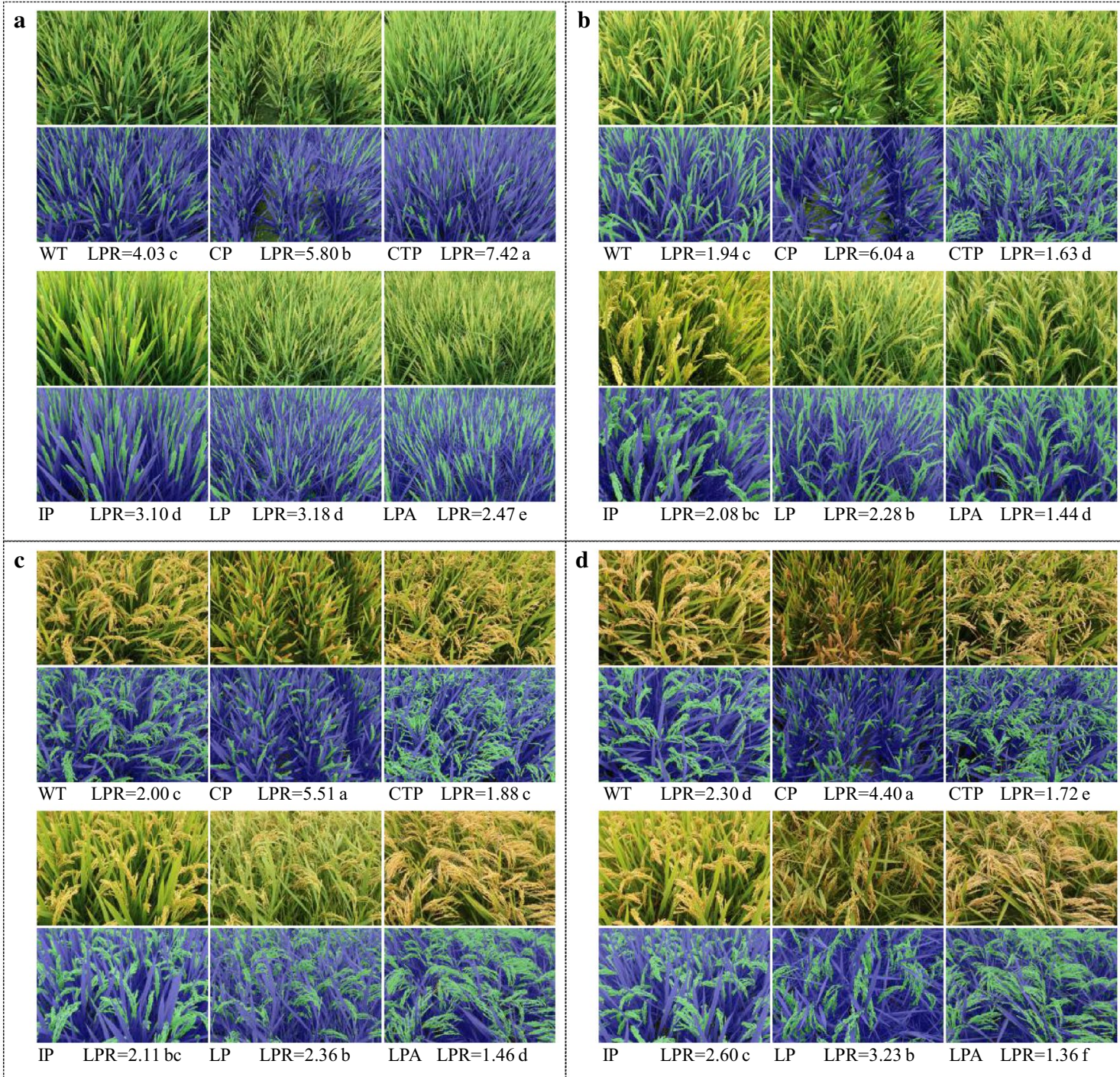

Fig. 8 LPRs of cultivars with six panicle types at different growth stages. a 0 DAA (days after anthesis); b 15 DAA; c 30 DAA; d 45 DAA. WT wild type, CP compact panicle, CTP chicken toe panicle, IP intermediate panicle, LP loose panicle, LPA loose panicle with awns. The CTP is a special type of $L P$ with more secondary branches on the upper part of the axis, with the lower rachis branches being curved into different directions similar to a chicken foot

semantic segmentation method, manual modification of auto-segmentation result function and export of LPR report function.

This work represents a proof of concept that deep learning can be used for accurate organ level (panicle, leaf) pixel-wise segmentation of field images. The LPR proposed is a novel trait in plant biology, and it is the deep learning method that make it possible to be detected and estimated. Deep learning has been applied to segment plant organs like leaf [15-18] and spike/panicle [19-24], with the aim of counting the number of them and thereby estimating crop yield. This study is totally different from the previous ones, because the newly developed trait of LPR here is based on simultaneous segmentation of leaf and panicle, neither counting the number nor separately detecting them. However, there are several challenges to be tackled in the future.

First, segmentation accuracy was quite high for these 6 GB datasets, but if objects were not included in the training dataset, it would have not performed as well. In other words, the robustness of a deep learning model is partially dependent on the diversity of the training dataset. In the future, we will seek to improve the robustness of FPN-Mask by collecting a wider range of field data. 


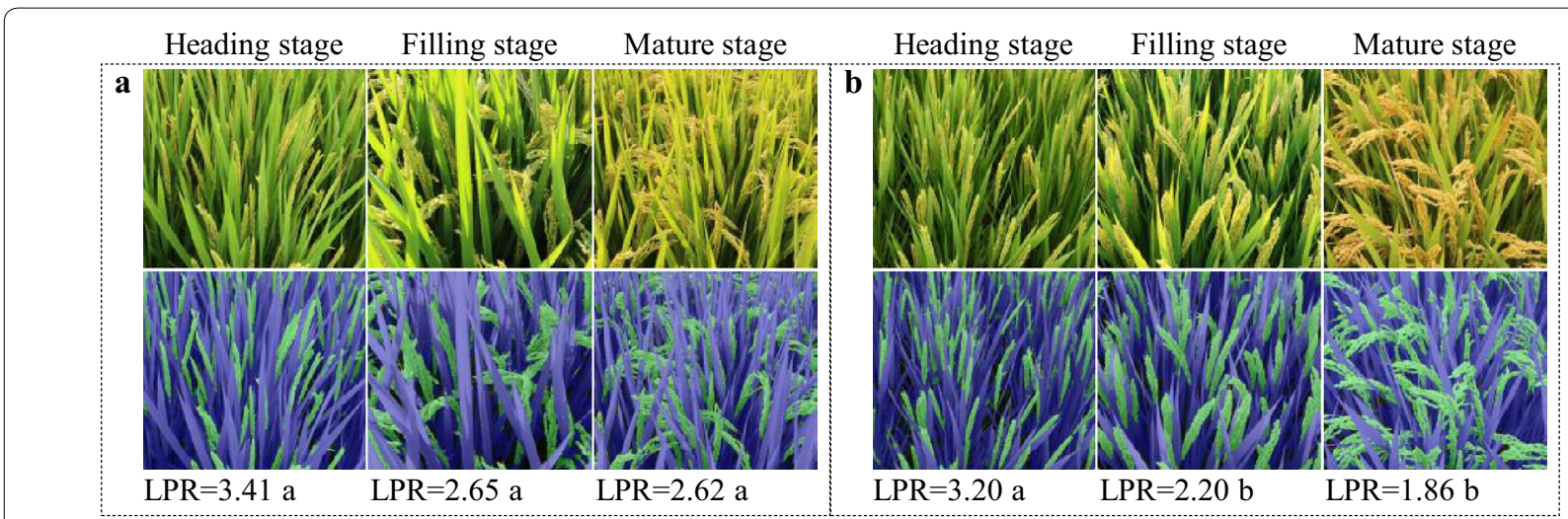

Fig. 9 Effect of $\mathrm{N}$ fertilization modes on LPR during grain filling. Japonica rice cultivar Wuyunjing 30 is used for representation. A, N5-5, N fertilization treatment with a base/topdressing ratio of 5:5; B, N treatment of N10-0

Second, the shadow on leaves and background exhibited very similar visual patterns. It is difficult to distinguish red, green and blue in the visible band. The junctions between different parts of plants are also quite difficult to distinguish. This explained most of the low precision for the semantic segmentation, and these types of errors occurred in every image in the testing dataset. Other studies also encountered the same problem [18].

Third, perspective photography can cause the deformation of objects projected into 2D images, which in turn affects the accuracy of LPR. However, our method has an advantage of reliably calculating the relative value of leaf to panicle ratio using 2D photos, on which the leaf and panicle in the 3D stand is compressed proportionally according to the imaging principle of the camera. Recently, light detection and ranging (LiDAR) has shown its advantages for showing high resolution 3-dimensional (3D) structural information of terrain and vegetation [42-44] and the advantage for segmentation of plant organs [16, 45, 46]. Shi et al. [18] also showed that a multi-view 3D system can avoid these errors. In the future, we will combine plant height provided by LiDAR to texture and color information provided by the RGB image to distinguish object categories more effectively and accurately.

\section{Significance of LPR for crop breeding and management}

To some degree, the essence of crop sciences is the knowledge of selection (by breeders) or regulation (by agronomists) of agronomical traits. Traditionally, crop scientists heavily depend on visual inspection of crops in the field as well as their evaluation of target traits based on their experience and expertise of the crop, which is labor intensive, time consuming, relatively subjective, and prone to errors [14, 47]. In addition, the target traits are mainly morphological traits including leaf senescence, plant height, tillering capacity, panicle or spike size, and growth periods, while fewer physiological traits are monitored and analyzed. With the development of plant phenotyping techniques, image-based methods have been successfully applied to obtain phenotypic data related to crop morphology and physiology [16]. In wheat, high throughput methods for a large array of traits are available for the breeders, including canopy temperature, normalized difference vegetation index (NDVI), and chlorophyll fluorescence [48]. However, the capacity for undertaking precision phenotyping of physiological traits is lagging far behind the requirement of crop sciences.

In this study, we propose a new physiological trait, LPR, based on deep learning. Physiologically, LPR indicates the distribution of light interception within the canopy between the source organ leaf and the sink organ panicle. Historically, breeders and agronomists focused on improvement in source activity, with traits of the leaf such as photosynthesis, erectness, and stay-green as the main targets. On the other hand, the role of panicle was largely overlooked, with less attention except for grain number per panicle or erectness of the panicle [7]. The significance of the panicle has been increasingly recognized in terms of its substantial contribution of carbohydrates, nitrogen, and minerals to grain filling. Therefore, light interception of panicles is dispensable for yield formation, and there should be a suitable LPR value for a crop stand growing in a given ecological condition.

The trait of LPR should provide crop scientists with new insights into the physiological status of the crop stand from the perspective of source and sink balance. For breeders, large genotypic variations in LPR are detected among the 192 germplasms, with a range of 1.37 to 5.60 , indicating the possibility to select elite parents for target hybridization and future studies on the morphological and physiological foundations of LPR. 


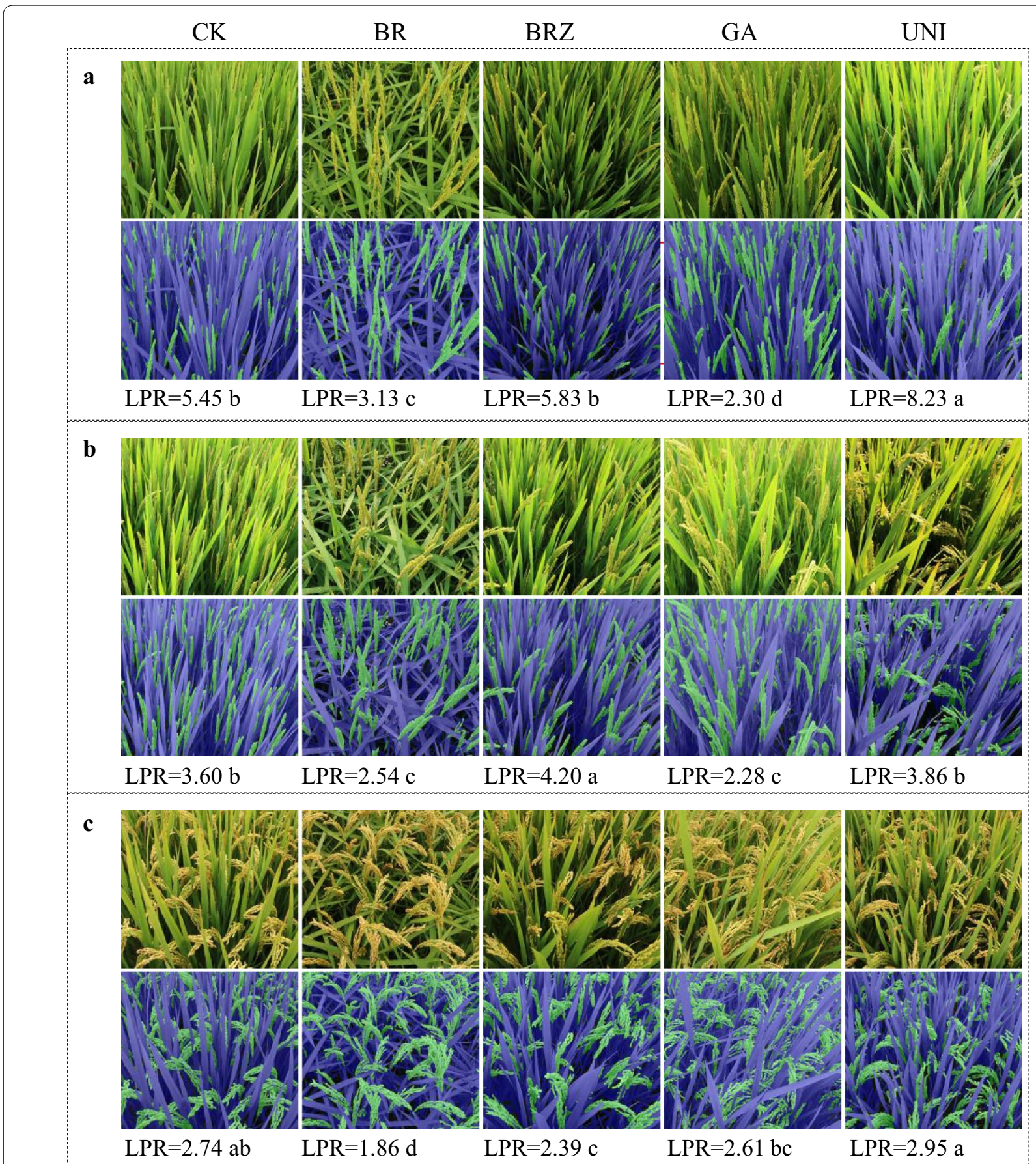

Fig. 10 Response of LPR to plant growth regulators. Ningjing 8 is used for representation. a Heading stage; $\mathbf{b}$ grain filling stage; $\mathbf{c}$ mature stage. CK, water spraying; BR, brassinolide; BRZ, brassinazole; GA, gibberellin; UNI, uniconazole

For agronomists, LPR is affected by nitrogen fertilization mode, and high yielding practice of N5-5 showed a relatively higher LPR value than that of N10-0, explaining the yield promotion effect of nitrogen topdressing in terms of source-sink relations. Further, LPR was sensitive to foliar application of plant growth regulators like BR and
GA, and can be increased by brassinazole and uniconazole, or reduced by brassinolide and gibberellin. Thus it is possible to develop methods for targeted regulation of crop stands with a desirable LPR by chemical intervention. In addition, LPR can be easily measured by digital camera and even a smartphone camera (data not shown). 
Considering that the measurement of LPR is vulnerable to variations in lighting conditions, we are currently conducting an experiment to identify the timing that can represent the average or general value of LPR for the whole daytime, which could facilitate the use of LPR by crop scientists. Nevertheless, more work is needed when applying LPR in crop breeding or management, in particular elucidating the inherent link between LPR and yield, and proposing a set of suitable LPR values for different environments or plant types.

\section{Conclusion}

The work represents a proof of the concept that the deep learning can achieve high accuracy in simultaneously detecting panicle and leaf data from complex rice field images. The FPN-Mask model is applicable for detecting and quantifying crop performance under field conditions. The proposed trait of LPR displayed large spatial and temporal variations as well as genotypic differences. It was also sensitive to agronomical practices such as nitrogen fertilization and spraying of plant growth regulators. Therefore, LPR, the novel trait indicative of source and sink relation, should provide a high throughput protocol for breeders to select superior rice cultivars as well as for agronomists to precisely manage field crops to have a good balance of source and sink. However, there are several challenges to be handled in future work, in particular combining plant height by LiDAR with the texture and color information from RGB image to distinguish object categories more effectively and accurately.

\section{Supplementary information}

Supplementary information accompanies this paper at https://doi. org/10.1186/s13007-020-00660-y.

Additional file 1. Additional figures and tables.

\begin{abstract}
Abbreviations
BR: Brassinolide; BRZ: Brassinazole; CP: Compact panicle; CT: Chicken toe; CTP: Chicken toe panicle; 3D: 3-Dimensional; 2D: 2-Dimensional; DAA: Days after anthesis; DCLISD: Dynamic canopy light interception simulating device; EP: Erect panicle; FPN: Feature pyramid network; FPN-mask: Feature pyramid network mask; GA: Gibberellin; GG: Green panicle with green leaf; GPU: geForce graphics processing unit; loU: Intersection-over-union; IP: Intermediate panicle; L: Leaf; LiDAR: Light detection and ranging; LP: Loose panicle; LPA: Loose panicle with awns; LPR: Leaf to panicle ratio; Max: Maximum; Min: Minimum; mloU: Mean intersection-over-union; N: Nitrogen; NDVI: NORMALIZED difference vegetation index; N5-5: N fertilization mode with base/topdressing ratio of 5/5; N10-0: N fertilization mode with base/topdressing ratio of 10/0; Panicle; PA: PIXEL accuracy; ResNet: Residual Networks; RGB: Red-green-blue; ROC: Receiver operating characteristic; ROI: Region of interest; SLIC: Simple linear iterative clustering; Std: Standard error of mean; UNI: Uniconazole; WT: Wild type; YG: Yellow panicle with green leaf; YY: Yellow panicle with yellow leaf.
\end{abstract}

\section{Acknowledgements}

Not applicable.

\section{Authors' contributions}

ZY and SG had the main responsibility for data collection and analysis, FX contributed to data collection and analysis, GL, YD, and MP revised the manuscript, QG and ZL (the corresponding authors) had the overall responsibility for experimental design, project management and manuscript preparation. All authors read and approved the final manuscript.

\section{Funding}

The research was supported by the National Key R\&D Program, Ministry of Science and Technology, China (2017YFD0300103), the National Natural Science Foundation of China (31771719), and National High Technology Research and Development Program of China (2014AA10A605). Rothamsted Research receives strategic funding from the Biological and Biotechnological Sciences Research Council of the United Kingdom. Matthew Paul acknowledges the Designing Future Wheat Strategic Programme (BB/P016855/1).

\section{Availability of data and materials}

The data used in this study is available from the corresponding author on reasonable request.

\section{Ethics approval and consent to participate}

Not applicable.

\section{Consent for publication}

Not applicable.

\section{Competing interests}

The authors declare that they have no conflicts of interest.

\section{Author details}

${ }^{1}$ College of Agriculture, Nanjing Agricultural University, Nanjing 210095, China. ${ }^{2}$ State Key Laboratory of Vegetation and Environmental Change, Institute of Botany, Chinese Academy of Sciences, Beijing 100093, China.

${ }^{3}$ Plant Science, Rothamsted Research, Harpenden, Hertfordshire AL5 2JQ, UK. ${ }^{4}$ Collaborative Innovation Center for Modern Crop Production, Nanjing Agricultural University, Nanjing 210095, China.

Received: 23 April 2020 Accepted: 18 August 2020

Published online: 26 August 2020

\section{References}

1. FAO, IFAD, UNICEF, WFP, WHO, The state of food security and nutrition in the world 2019. Safeguarding against economic slowdowns and downturns, Rome. 2019. https://www.wfp.org/publications/2019-state -food-security-and-nutrition-world-sofi-safeguarding-against-economic.

2. Varshney RK, Sinha P, Singh VK, Kumar A, Zhang QF, Bennetzen JL. 5Gs for crop genetic improvement. Curr Opin Plant Biol. 2019;13:1-7. https://doi. org/10.1016/j.pbi.2019.12.004.

3. Großkinsky DK, Svensgaard J, Christensen S, Roitsch T. Plant phenomics and the need for physiological phenotyping across scales to narrow the genotype-to-phenotype knowledge gap. J Exp Bot. 2015;66(18):5429-40. https://doi.org/10.1093/jxb/erv345.

4. Reynolds M, Langridge P. Physiological breeding. Curr Opin Plant Biol. 2016;31:162-71. https://doi.org/10.1016/j.pbi.2016.04.005.

5. Xie F, Hardy B. Accelerating hybrid rice development. Los Baños (Philippines): International Rice Research Institute; 2009.

6. Wang B, Smith SM, Li JY. Genetic regulation of shoot architecture. Annu Rev Plant Biol. 2018;69:437-68. https://doi.org/10.1 146/annurev-arpla nt-042817-040422.

7. Qian Q, Guo LB, Smith SM, Li JY. Breeding high-yield superior quality hybrid super rice by rational design. Natl Sci Rev. 2016;3(3):283-94. https ://doi.org/10.1093/nsr/nww006.

8. Yang JC, Zhang JH. Grain-filling problem in 'super' rice. J Exp Bot. 2010;61(1):1-4. https://doi.org/10.1093/jxb/erp348.

9. Paul MJ, Oszzald M, Jesus C, Rajulu C, Griffiths CA. Increasing crop yield and resilience with trehalose 6-phosphate: targeting a feast-famine mechanism in cereals for better source-sink optimization. J Exp Bot. 2017;68(16):4455-62. https://doi.org/10.1093/jxb/er×083. 
10. Zhang XC, Lei JC, Zheng DY, Liu ZH, Li GH, Wang SH, Ding YF. Amino acid composition of leaf, grain and bracts of japonica rice (Oryza Sativa ssp. japonica) and its response to nitrogen fertilization. Plant Growth Regul. 2017;82(1):1-9. https://doi.org/10.1007/s10725-016-0232-5.

11. Wang ZX, Zhang FF, Xiao F, Tao Y, Liu ZH, Li GH, Wang SH, Ding YF. Contribution of mineral nutrients from source to sink organs in rice under different nitrogen fertilization. Plant Growth Regul. 2018;86(2):159-67. https://doi.org/10.1007/s10725-018-0418-0.

12. Sanchez-Bragado R, Molero G, Reynolds MP, Araus JL. Photosynthetic contribution of the ear to grain filling in wheat: a comparison of different methodologies for evaluation. J Exp Bot. 2016;67(9):2787-98. https://doi. org/10.1093/jxb/erw116.

13. Imairumi N, Usuda H, Nakamoto H, Ishihara K. Changes in the rate of photosynthesis during grain filling and the enzymatic activities associated with the photosynthetic carbon metabolism in rice panicles. Plant Cell Physiol. 1990;31(6):835-44. https://doi.org/10.1093/oxfordjournals.pcp. a077986.

14. Yang WN, Feng H, Zhang XH, Zhang J, John HD, Batchelor WD, Xiong LZ, Yan JB. Crop phenomics and high-throughput phenotyping: past decades, current challenges, and future perspectives. Mol Plant. 2020;13(2):187-21414. https://doi.org/10.1016/j.molp.2020.01.008.

15. Aakif A, Khan MF. Automatic classification of plants based on their leaves. Biosyst Eng. 2015;139:66-75. https://doi.org/10.1016/j.biosystems eng.2015.08.003.

16. Jin SC, Su YJ, Gao S, Wu FF, Hu TY, Liu J, Li WK, Wang DC, Chen SJ, Jiang YJ, Pang SX, Guo QH. Deep Learning: individual maize segmentation from terrestrial LiDAR data using faster R-CNN and regional growth algorithms. Front Plant Sci. 2018;9:866. https://doi.org/10.3389/fpls.2018.00866.

17. Ubbens J, Cieslak M, Prusinkiewicz P, Stavness L. The use of plant models in deep learning: an application to leaf counting in rosette plants. Plant Methods. 2018;14:6. https://doi.org/10.1186/s13007-018-0273-z.

18. Shi WN, Zedde RVD, Jiang HY, Kootstra G. Plant-part segmentation using deep learning and multi-view vision. Biosyst Eng. 2019;187:81-95. https:// doi.org/10.1016/j.biosystemseng.2019.08.014.

19. Duan $L F$, Huang $C L$, Chen $G X$, Xiong LZ, Liu Q, Yang WN. Determination of rice panicle numbers during heading by multi-angle imaging. Crop $\mathrm{J}$. 2015;3(3):211-9. https://doi.org/10.1016/j.cj.2015.03.002.

20. Xiong X, Duan LF, Liu LB, Tu HF, Yang P, Wu D, Chen GX, Xiong LZ, Yang WN, Liu Q. Panicle-SEG: a robust image segmentation method for rice panicles in the field based on deep learning and superpixel optimization. Plant Methods. 2017;13:104. https://doi.org/10.1186/s13007-017-0254-7.

21. Desai SV, Balasubramanian VN, Fukatsu T, Ninomiya S, Guo W. Automatic estimation of heading date of paddy rice using deep learning. Plant Methods. 2019;15:76. https://doi.org/10.1186/s13007-019-0457-1.

22. Hasan MM, Chopin JP, Laga H, Miklavcic SJ. Detection and analysis of wheat spikes using Convolutional Neural Networks. Plant Methods. 2018;14:100. https://doi.org/10.1186/s13007-018-0366-8.

23. Fernandez-Gallego JA, Kefauver SC, Gutiérrez NA, Nieto-Taladriz MT, Araus $J$. Wheat ear counting in-field conditions: high throughput and low-cost approach using RGB images. Plant Methods. 2018;14:22. https://doi. org/10.1186/s13007-018-0289-4.

24. Pouria ST, Virlet N, Ampe EM, Reyns P, Hawkesford MJ. DeepCount: in-field automatic quantification of wheat spikes using simple linear iterative clustering and deep convolutional neural networks. Front Plant Sci. 2019;10:1176. https://doi.org/10.3389/fpls.2019.01176.

25. Lin TY, Dollár P, Girshick RB, He K, Hariharan B, Belongie SJ. Feature pyramid networks for object detection. Proceedings of the IEEE conference on computer vision and pattern recognition. 2017;1:2117-255. https:// doi.org/10.1109/CVPR.2017.106.

26. Abacar JD, Lin ZM, Zhang XC, Ding CQ, Tang S, Liu ZH, Wang SH, Ding YF. Variation in yield and physicochemical quality traits among mutants of japonica rice cultivar Wuyujing 3. Rice Sci. 2016;23(1):33-41. https://doi. org/10.1016/j.rsci.2016.01.004.

27. Zhang XC, Alim MA, Lin ZM, Liu ZH, Li GH, Wang QS, Wang SH, Ding YF. Analysis of variations in white-belly and white-core rice kernels within a panicle and the effect of panicle type. J Integr Agric. 2014;13(8):1672-9. https://doi.org/10.1016/S2095-3119(13)60593-6.

28. Santurkar S, Tsipras D, llyas A, Madry A. How does batch normalization help optimization? In: Proceedings of the 32nd international conference on neural information processing systems. 2018, p. 2488-2498.
29. Ioffe S, Szegedy C. Batch normalization: accelerating deep network training by reducing internal covariate shift. In: Proceedings of the 32 nd international conference on machine learning. 2015, p. 37:448-456.

30. Xiao T, Liu YC, Zhou B, Jiang YN, Sun J. Unified perceptual parsing for scene understanding. Proc Eur Conf Comput Vis. 2018;1:432-48. https:// doi.org/10.1007/978-3-030-01228-1_26.

31. He KM, Zhang XY, Ren SQ, Sun J. Deep residual learning for image recognition. In: Proceedings of the IEEE conference on computer vision and pattern recognition. 2016, p. 770-778. https://doi.org/10.1109/ CVPR.2016.90.

32. Zhao HS, Shi JP, Qi XJ, Wang XG, Jia JY. Pyramid scene parsing network. In: Proceedings of the IEEE conference on computer vision and pattern recognition. 2017, p. 6230-6239. https://doi.org/10.1109/CVPR.2017.660.

33. Krizhevsky A, Sutskever I, Hinton GE. Imagenet classification with deep convolutional neural networks. Commun ACM. 2017;60:6. https://doi. org/10.1145/3065386.

34. Lin TY, Goyal P, Girshick R, He K, Dollár P. Focal loss for dense object detection. IEEE Trans Pattern Anal Mach Intell. 2017;1:318-27. https://doi. org/10.1109/TPAMI.2018.2858826.

35. He KM, Zhang XY, Ren SQ, Sun J. Delving deep into rectifiers: Surpassing human-level performance on imagenet classification. In: Proceedings of the IEEE international conference on computer vision. 2015, p. 1026-1034. https://doi.org/10.1109/ICCV.2015.123.

36. Ren CY, Prisacariu VA, Reid ID. gSLICr: SLIC superpixels at over $250 \mathrm{~Hz}$. arXiv e-prints. 2015.

37. Achanta R, Shaji A, Smith K, Lucchi A, Fua P, Süsstrunk S. SLIC superpixels compared to state-of-the-art superpixel methods. IEEE Trans Pattern Anal Mach Intell. 2012;34(11):2274-81. https://doi.org/10.1109/TPAMI 2012.120.

38. Hanley JA, Mcneil BJ. The meaning and use of the area under a receiver operating characteristic (ROC) curve. Radiology. 1982;143(1):29-36. https ://doi.org/10.1148/radiology.143.1.7063747.

39. Sun SY, Chen DH, Li XM, Li CX, Shen HY, Wang XL. Brassinosteroid signaling regulates leaf erectness in Oryza sativa via the control of a specific U-Type cyclin and cell proliferation. Dev Cell. 2015;34:220-8. https://doi. org/10.1016/j.devcel.2015.05.019.

40. Tong HN, Chu CG. Functional specificities of brassinosteroid and potential utilization for crop improvement. Trends Plant Sci. 2018;23(11):1016-28. https://doi.org/10.1016/j.tplants.2018.08.007.

41. Zhu YY, Nomura T, Xu YH, Zhang YY, Peng Y, Mao BZ, Hanada A, Zhou HC, Wang RX, Li PJ, Zhu XD, Mander LN, Kamiya YJ, Yamnguchi S, He ZH. Elongated Uppermost Internode encodes a cytochrome P450 monooxygenase that epoxidizes gibberellins in a novel deactivation reaction in rice. Plant Cell. 2006;18(2):442-56. https://doi.org/10.1105/tpc.105.038455.

42. Guo QH, Liu J, Tao SL, Xue BL, Li L, Xu GC, Li WK, Wu FF, Li YM, Chen $L H$, Pang SX. Perspectives and prospects of LiDAR in forest ecosystem monitoring and modeling. Chin Sci Bull. 2014;59(6):459-78. https://doi. org/10.1360/972013-592.

43. Lefsky MA, Cohen WB, Parker GG, Harding DJ. LiDAR remote sensing for ecosystem studies LiDAR, an emerging remote sensing technology that directly measures the three-dimensional distribution of plant canopies, can accurately estimate vegetation structural attributes and should be of particular interest to forest, landscape, and global ecologists. Psychol Rep. 2002;52(1):19-30. https://doi.org/10.1641/00063568(2002)052[0019:LRSFES]2.0.CO;2.

44. LiYM, Guo QH, Su YJ, Tao SL, Zhao KG, Xu GC. Retrieving the gap fraction, element clumping index, and leaf area index of individual trees using single-scan data from a terrestrial laser scanner. ISPRS J Photogramm Remote Sens. 2017;130:308-16. https://doi.org/10.1016/j.isprs jprs.2017.06.006.

45. Jin SC, Su YJ, Wu FF, Pang SX, Gao S, Hu TY, Liu J, Guo QH. Stem-Leaf segmentation and phenotypic trait extraction of individual maize using terrestrial LiDAR data. IEEE Trans Geosci Remote Sensing. 2019;57(3):133646. https://doi.org/10.1109/TGRS.2018.2866056.

46. Jin SC, Su YJ, Gao S, Wu FF, Ma Q, Xu KX, Hu TY, Liu J, Pang SX, Guan HC, Zhang J, Guo QH. Separating the structural components of maize for field phenotyping using terrestrial LiDAR data and deep convolutional neural networks. IEEE Trans Geosci Remote Sensing. 2020;58(4):2644-58. https:// doi.org/10.1109/TGRS.2019.2953092.

47. Alkhudaydi T, Reynolds D, Griffiths S, Zhou J, Iglesia Bl. An exploration of deep-learning based phenotypic analysis to detect spike regions 
in field conditions for UK bread wheat. Plant Phenomics. https://doi. org/10.34133/2019/7368761.

48. Pask AJD, Pietragalla J, Mullan DM, Reynolds MP. Physiological breeding II: a field guide to wheat phenotyping. CIMMYT. 2012. https://hdl.handl e.net/10883/1288.

\section{Publisher's Note}

Springer Nature remains neutral with regard to jurisdictional claims in published maps and institutional affiliations.
Ready to submit your research? Choose BMC and benefit from:

- fast, convenient online submission

- thorough peer review by experienced researchers in your field

- rapid publication on acceptance

- support for research data, including large and complex data types

- gold Open Access which fosters wider collaboration and increased citations

- maximum visibility for your research: over 100M website views per year

At BMC, research is always in progress.

Learn more biomedcentral.com/submissions 